\title{
Research on Parameter Identification of Closed Loop Control System of Electromagnetic Speed Regulation Coal Cutter
}

\author{
Mingxing $\mathrm{He}^{1, \mathrm{a}}$ \\ ${ }^{1}$ China University of Mining and Technology (Beijing) School of Mechanical, Electrical \\ Information Engineering, Beijing 100083 \\ ahmx@accshow.com
}

\begin{abstract}
Keywords: Relay feedback Control; Electromagnetic Speed Regulation Coal Cutter; Parameter Identification; Double Closed Loop
\end{abstract}

\begin{abstract}
Electromagnetic speed regulation coal cutter has simple structure and high performance cost ratio, and the PID or improved PID controller can enable stable and high efficiency operation of coal cutter. Since the underground coal mine has complex and uncertain operation condition, the coal cutter during actual operation may not reach the predicted performance requirement, controller parameter shall be adjusted. Electromagnetic speed regulation coal cutter system parameter identification method, on the basis of relay feedback self-tuning, is proposed, and relay cascade feedback control is introduced to respectively online optimize control parameter of speed loop and current loop, update controller parameter, and evaluate system response performance. The simulation and test result verifies the effectiveness of parameter identification result of the method, and improves the anti-jamming capability of the control system.
\end{abstract}

\section{Introduction}

The coal cutter is the critical electric equipment for coal mine production. At present, the domestic coal cutter almost all adopts electric traction mode. According to the traction motor type, it can be divided into variable frequency drive, switch reluctance drive and electromagnetic speed regulation types, wherein, electromagnetic speed regulation coal cutter has stable operation, low cost and easy maintenance with certain position among coal cutter driving. Electromagnetic speed regulation motor is also called slop frequency motor, the slip frequency clutch regulates the excitation current to change magnetic flux and realize energy exchange and AC speed control. The control mode is usually PID closed loop control to regulate rotation speed output of motor. Literature 1 analyzed the operation principle of double closed loop of electromagnetic speed regulation motor, and manual experience modification method was adopted to regulate system PID parameter with fairly low efficiency; Literature 2 adopted mathematical model structure of current loop and speed loop in case of double closed loop control, but there was no analysis and simulation for the system parameter; Literature 3 studied the operation condition characteristic of electromagnetic speed regulation coal cutter brake operation, and analyzed the impact of coal cutter system parameter to brake performance, but there was no quantitative analysis by using theoretical method; Literature 4 pointed out the problems of simulation PID regulator parameter drifting and commissioning of electromagnetic speed regulation motor, and adopted maximum membership degree ambiguity resolution controller real time regulation and simulation curve to present that, fuzzy controller control was more unblocked, but compilation of fuzzy rule also relied on manual offline resolution 
and could not realize self-adaptation online identification. Aiming at complicated operation condition characteristics of electromagnetic speed regulation coal cutter, this paper proposes the relay feedback based online parameter identification method, adopts the cascade feedback to enable coordinated operation of double closed loop, and there is no impact to normal operation of system during the parameter identification, so PID tuning parameter can be updated online and real time.

\section{Parameter Tuning Method of Control System of Electromagnetic Speed Regulation Coal Cutter}

Structure Characteristic and Control Mode. The electromagnetic speed regulation motor is composed of two parts as drive and driven, as shown in Fig. 1.

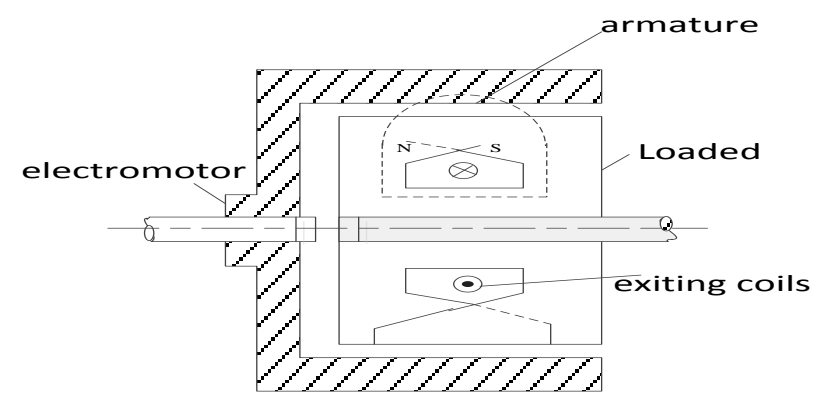

Fig. 1 Sructure of Magnetic Slip Clutch

The drive part is connected with one ordinary squirrel cage asynchronous motor, the driven part is connected with traction load of coal cutter, and two parts are connected by slip frequency clutch. The slip frequency clutch consists of armature and exciting winding, in the exciting winding, DC is connected to create an alternating magnetic field, rotation of prime motor drives the rotation of armature to cut the magnetic line of force, and drive load of driven shaft, to realize AC speed regulation.

Energy of primer motor and load are completed via magnetic exchange, magnetic permeability of excitation clutch is closely related to mechanical construction, environment and rotation speed, the natural mechanical property is fairly weak, in order to enable stable operation of electromagnetic speed regulation motor, the manual mechanical property curve shall substitute the natural property so that it is approaching to linear relation within the regulation range, as shown in Fig. 2.
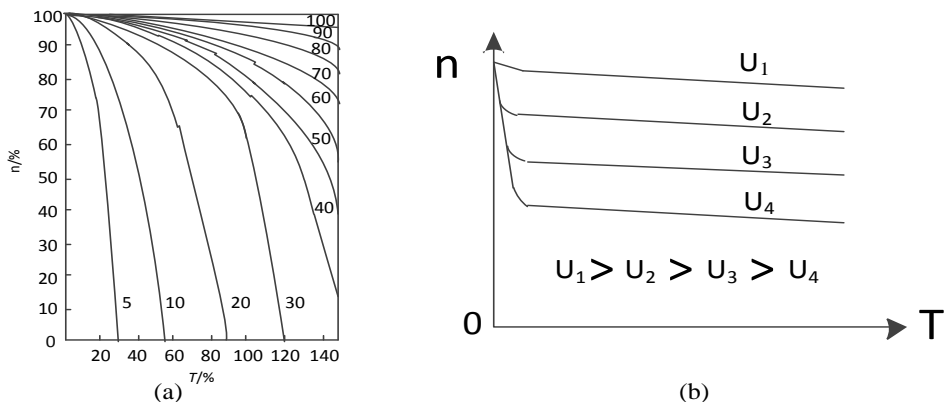

(b)

Fig. 2 The Natural and Artificial Characteristic Curve of Slip Motor 
Among the motor controls, PID controller is usually adopted to supervise the error between motor output speed and set speed. According to errors, the output quantity is regulated to change speed. Underground operation of electromagnetic speed regulation coal cutter is usually disturbed by fluctuation of grid and current caused by startup/shutdown of other big power equipment, and this incurs delay of speed feedback control. In order to improve control quality, current loop PID controller is introduced in, when there is a sudden change of exciting current, and it is conducted by suppression regulation to ensure the system stability, as shown in Fig. 3. The control system of double closed loop electromagnetic speed regulation coal cutter has the advantages of quick response speed and high regulation accuracy, system parameter of two closed loop controller has critical impact to performance of whole system.

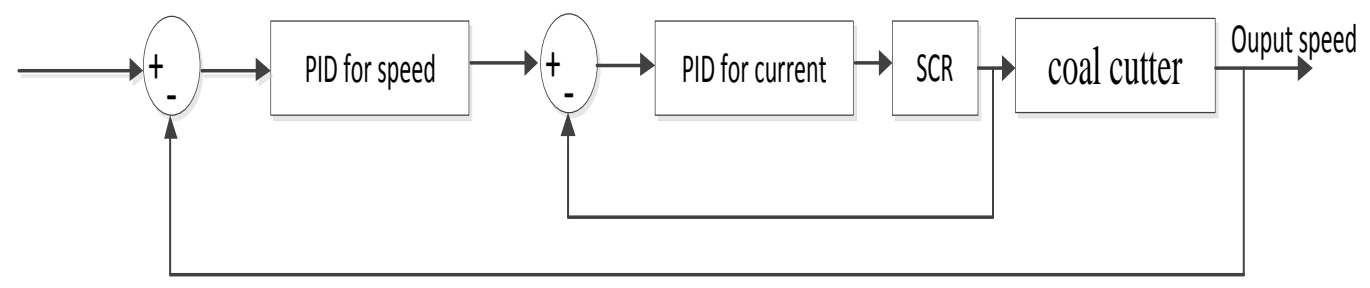

Fig. 3 Double Closed Loop Diagram of Electromagnetic Speed of Shearer

\section{Design of Electromagnetic Speed Regulation Relay Feedback Algorithm}

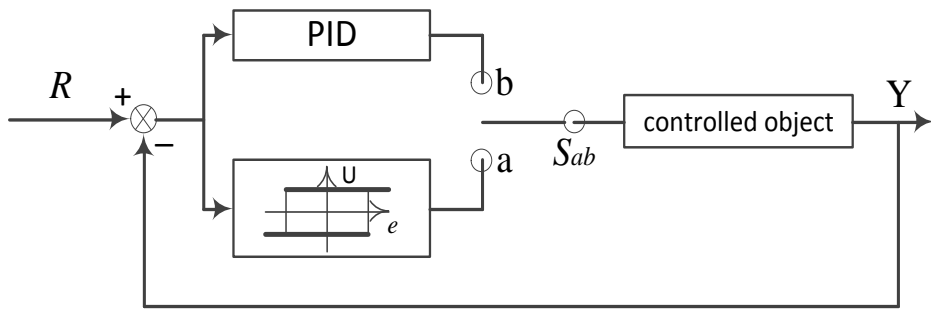

Fig. 4 Self-tuning Relay Schematic Diagram

Theory of relay feedback. The structural chart of relay self-tuning parameter is shown in Fig. 4. The tuning equipment consists of un-regulated PID controller, controlled object and ideal relay element. When single-pole double-throw switch $\mathrm{S}$ and a point are closed, the system shall start the relay feedback self-tuning test process. The hysteresis of system shall cause same amplitude oscillation to derive critical gain $\mathrm{K}_{\mathrm{u}}$ and critical cycle $\mathrm{T}_{\mathrm{u}}$, and calculate PID parameter. PID parameter is updated to PID controller, switch $\mathrm{S}$ and $\mathrm{b}$ points are closed, the system adopts the controller (after parameter update) to regulate the controlled object.

The describing function method is adopted to conduct quantitative analysis to Fig. 4 . When the relay is hysteresis loop type, the describing function is:

$$
-\frac{1}{N(A)}=-\frac{\pi}{4 d}\left[\sqrt{A^{2}-\varepsilon^{2}}+j \varepsilon\right]
$$


Wherein, $\mathrm{A}$ is fundamental current amplitude, $\mathrm{d}$ is loop back amplitude value, $\varepsilon$ is loop back threshold. Assume that transfer function of controlled object is $G_{p}(s)$, for occurrence of system oscillation at tuning mode, the following shall be acquired:

$$
G_{p}(j w)=-\frac{1}{N(A)}
$$

According to formula (1) and (2), critical gain of system is:

$$
K_{\mathrm{u}}=|N(A)|=\frac{4 d}{\pi A}
$$

Two neighboring zero points or extremities are taken on stable and even oscillation cycle, assume that the frequency is $w_{c}$, the critical cycle is:

$$
\mathrm{T}_{\mathrm{u}}=\mathrm{T}_{\mathrm{c}}=\frac{2 \pi}{\mathrm{w}_{\mathrm{c}}}
$$

Determination of controller structure and system function of electromagnetic speed regulation coal cutter. According to the construction characteristic of electromagnetic coal cutter and armature circuit equation, the transfer function of slip frequency clutch of electromagnetic speed regulation coal cutter can be approx. presented as:

$$
W_{d}(s)=\frac{1}{R} \cdot \frac{1}{1+K_{\mathrm{d}} S}
$$

Wherein, $\mathrm{R}$ is the resistance of armature circuit, $\mathrm{K}_{\mathrm{d}}$ is the time constant of electromagnetic motor, and the value is the proportion between armature circuit resistance and inductance. The transfer function of motor is:

$$
W_{\mathrm{j}}(\mathrm{S})=\frac{\mathrm{K}_{1}}{\mathrm{~K}_{2}} \cdot \frac{1}{1+J / \mathrm{K}_{2} S}
$$

$\mathrm{J}$ is flywheel torque of motor, $\mathrm{K}_{1}$ is variation rate of exciting current, $\mathrm{K}_{2}$ is variation rate of electromagnetic torque, which are all related to mechanical characteristic of motor. The transfer function for rectification of thyristor current is:

$$
W_{\mathrm{k}}(S)=\frac{\mathrm{K}_{\mathrm{kb}}}{1+\tau_{\mathrm{s}} S}
$$

$\mathrm{K}_{\mathrm{kb}}$ is current gain amplification factor, $\tau_{\mathrm{s}}$ is current feedback time constant, according to Fig. 3, the rectification and motor transfer function can be acquired, as shown in Fig. 5: 


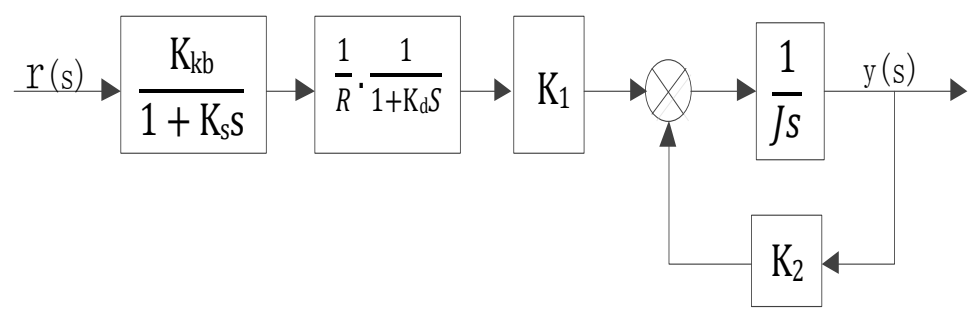

Fig. 5 Structure Diagram of the Rectifying and Motor Transfer Function

\section{Coal Cutter System Parameter Identification Process}

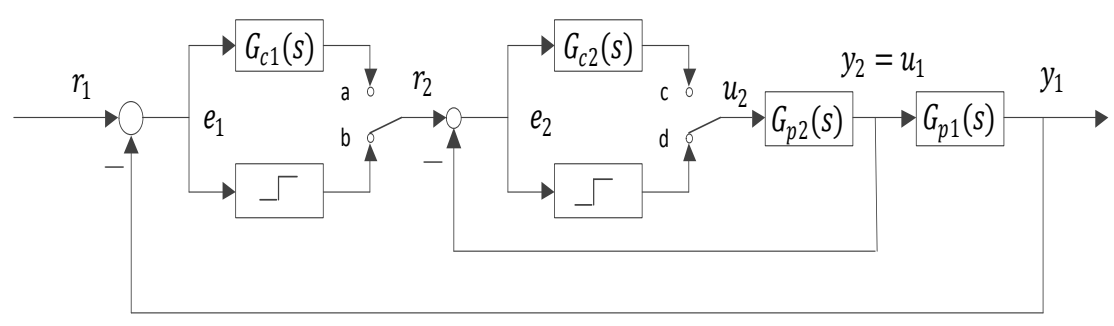

Fig. 6 Structure Diagram of Cascade Relay feedback Tuning

Electromagnetic speed regulation cascade double closed loop tuning. Modeling of electromagnetic speed regulation coal cutter tuning system is shown in Fig. 6. During the operation of electromagnetic speed regulation coal cutter, the frequency difference of inner loop and outer loop is fairly great, the speed regulation of outer loop is the main function of system, and the coupling degree of regulation effect is fairly low, the step-by-step tuning method can be adopted to respectively identify parameters of inner loop and outer loop, finally it is put into system operation. In the cascade structure, tuning sequence of inner loop and outer loop also has a critical impact to performance of controller. The tuning sequence can be analyzed by two viewpoints: (1) It is deemed as the extension of traditional feedback structure, and it shall be respectively calculated by switching branch during tuning process; (2) It is deemed as a multi-input and multi-output quantity system, the parallel structure is analyzed from the viewpoint of status equation. During the operation of electromagnetic speed regulation coal cutter, the manual tuning optimization may be required; feedback structure is adopted for test. The tuning work is divided into two steps, at first the branch switch is turned to a and d tuning current loop parameter, then it is put into inner loop operation, the switch is turned to b and c tuning outer loop parameter, and finally it is put into whole system. According to Fig. 5 and Fig. 6, the closed loop function of system during inner loop tuning can be:

$$
G(s)=\frac{G_{c 1}(s) G_{c 2}(s) G_{p 1}(s) G_{p 2}(s)}{1+G_{c 2}(s) G_{p 1}(s)\left(1+G_{c 1}(s) G_{p 1}(s)\right)}
$$

The critical gain of current loop of electromagnetic speed regulation coal cutter is:

$$
\mathrm{K}_{u 2}=\frac{4 d_{2}}{\pi a_{2}} \approx\left|\frac{-1}{G_{p 2(j \omega)}\left(1+G_{c 1(j \omega)} G_{p 1(j \omega)}\right)}\right|_{\omega=\omega_{u 2}}
$$


$G(s)=\frac{G_{c 1}(s) G_{p 1}(s)\left(\frac{G_{p 2(j \omega)} G_{c 2(j \omega)}}{1+G_{p 2(j \omega)} G_{c 2(j \omega)}}\right)}{1+G_{c 1}(s) G_{p 1}(s)\left(\frac{G_{p 2(j \omega)} G_{c 2(j \omega)}}{1+G_{p 2(j \omega)} G_{c 2(j \omega)}}\right)}$

During tuning of outer loop branch circuit, closed loop transfer function of system is:

$$
G(s)=\frac{G_{c 1}(s) G_{p 1}(s)\left(\frac{G_{p 2(j \omega)} G_{c 2(j \omega)}}{1+G_{p 2(j \omega)} G_{c 2(j \omega)}}\right)}{1+G_{c 1}(s) G_{p 1}(s)\left(\frac{G_{p 2(j \omega)} G_{c 2(j \omega)}}{1+G_{p 2(j \omega)} G_{c 2}(j \omega)}\right)}
$$

The critical gain of speed loop of electromagnetic speed regulation coal cutter is:

$$
\mathrm{K}_{u 1}=\frac{4 d_{1}}{\pi a_{1}} \approx\left|\frac{1}{G_{p 1(j \omega)}\left(\frac{G_{p 2(j \omega)} G_{c 2(j \omega)}}{1+G_{p 2(j \omega)} G_{c 2(j \omega)}}\right)}\right|_{\omega=\omega_{u 2}}
$$

According to formulas 8-11, the critical gain and critical frequency of inner and outer loop of electromagnetic speed regulation coal cutter system can be acquired.

Simulation and Test. Taking the electromagnetic speed regulation motor of Nanjing Shenma Brand as an example, its primary side armature circuit resistance is $\mathrm{R}=15 \Omega$, exciting current $\mathrm{I}=5 \mathrm{~A}$, thyristor amplification factor is $\mathrm{Kr}=40$, stator and rotor mutual inductance quantity is $2.5 \mathrm{~h}$, rotation inertia is $0.12 \mathrm{~kg} / \mathrm{m} 2$, rotation feedback coefficient $\alpha=0.05$, current feedback coefficient is $\beta=0.007$, rated rotation speed is $1360 \mathrm{r} / \mathrm{min}$. At Matlab environment, Simulink is adopted for simulation.

The relay test waveform of current inner loop is shown in Fig. 7, the amplitude value and frequency are read, according to computing of formula $3, \mathrm{~K}_{\mathrm{ui}}=0.13 \mathrm{~T}_{\mathrm{ui}}=54.51$; according to table $1, \mathrm{~K}_{\mathrm{pi}}=0.06 \mathrm{~T}_{\mathrm{ii}}=45.24$.

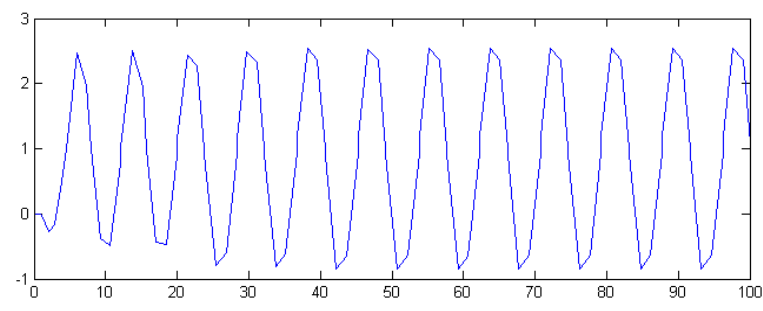

Fig. 7 The test diagram of current loop relay

The relay test waveform of speed outer loop is shown in Fig. 8, the amplitude value and frequency are read, according to computing of formula $3, \mathrm{~K}_{\mathrm{us}}=4.04 \mathrm{~T}_{\mathrm{us}}=0.29$; according to table 1 , $\mathrm{K}_{\mathrm{ps}}=4.04 \mathrm{~T}_{\mathrm{is}}=0.24$.

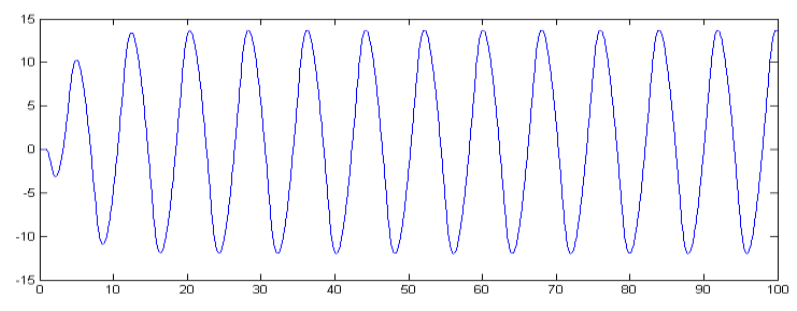

Fig. 8 The test diagram of speed loop relay 
After identification, the system step response and load disturbance simulation are shown in Fig. 9. The system over-shoot is $11 \%$, the steady status error is $0.6 \%$, stable time is $15.7 \mathrm{~s}$, and attenuation ratio is $48 \%$. After stabilization of system operation, at the first $60 \mathrm{~s}$, a disturbance of amplitude of 1.6 is added, the whole regulation process of disturbance is $11 \mathrm{~s}$, and the system over-shoot is $20 \%$, which fairly well plays the suppression effect.

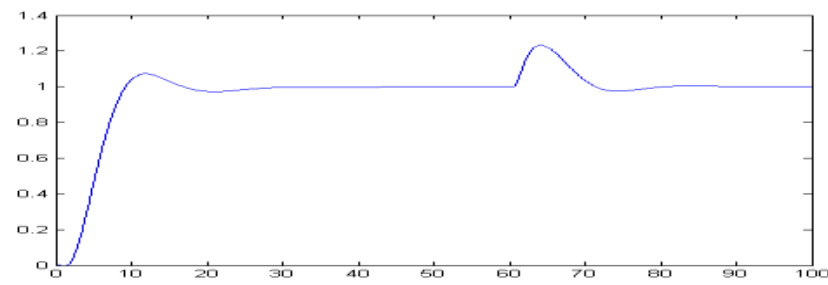

Fig. 9 The Step Response and Load Disturbance Diagram of Shearer

According to the model and simulation data of electromagnetic speed regulation coal cutter, embedded Linux is adopted to establish circuit and software control system, the main control chip is AT91SAM9260, operation speed is 270M, the system parameter after identification is written into control system. One dynamometer is adopted to add $95 \mathrm{~N} \cdot \mathrm{M}$ load to test characteristics of electromagnetic speed regulation coal cutter with load, the test result is shown in Fig. 10. The set speed of electromagnetic speed regulation coal cutter is 900 turns/min, the upward side is regulation quantity output curve, the middle position is process quantity motor rotation speed feedback curve, the regulation process is basically coincided with simulation result characteristic, and this proves effectiveness of identification result.

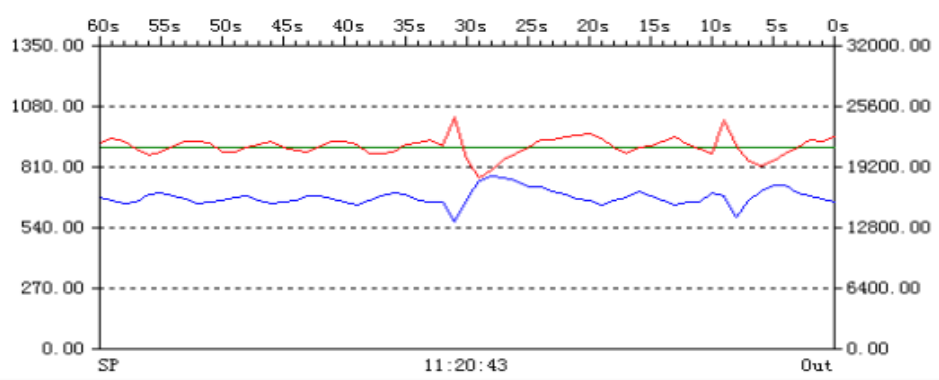

Fig. 10 The Load Characteristic of Electromagnetic Speed of Shearer

\section{Conclusion}

The relay feedback method is adopted to conduct the parameter identification to double closed loop control system of electromagnetic speed regulation coal cutter, the requirement over the object of system modeling is fairly low, and there are less experience for anticipation of operation condition, so the embedded system shall conduct the automatic identification in the steady status range of operation without impacting operation of previous system. It has the advantages of convenient use, quick computing speed and high output accuracy, and it is an effective method for inner and outer loop parameter tuning of electromagnetic speed regulation coal cutter. 


\section{References}

[1] LIU Jian-gong, WANG Ru-lin, LUO Wen-bin, The Study and Application of Computer Speed Regulating System of Electromagnetic Speed Regulating Electrical Haulage Shearer [J] Colliery Mechanical \& Electrical Technology, 2004,02:1-4.

[2] LIU Qian-Sheng, ZHANG Qiang-li, ZHANG Hong-ge. Design of the Electromagnetism Govern Electricity tow Mining Coal Machine Control System [J]. Hebei Coal, 2008, 06:8-9+54.

[3] LIU Xia, XIE Zj-dian Design of Electromagnetic Speed Regulation of Shearers Based on Fuzzy [J]. Control Industrial Control Computer, 2009, 05:55-56+58.

[4] LIU hai. Closed-Loop System Identification based on Relay Feedback and the Application in Model Predictive Control [D]. East China University of Science and Technology, 2013.

[5] LIU Jian-Gong. The Control System of Electromagnetic Speed Regulation of Shearers. Beijing: China Coal Industry Publishing House,2006.

[6] ZHANG Yi-Qun. Development of PID Auto tuning Software Package Based on Relay Feedback and Related Algorithms Study [D]. Northeastern University, 2005.

[7] Xiong Tao. Study Of The Algorithm Of Auto-Tuning PDF Control based On Relay Feedback [D].Southwest Jiaotong University, 2003

[8] Gu Dan-Ying. Research on Auto-Tuning Control Methods for Dead-Time System Based On Relay Feedback Technique [D]. Shanghai Jiao Tong University, 2010

[9] Ma Ming-Da, Zhu Xin-Jian, Survey in Relay Feedback Identification [J].Control and Instruments in Chemical Industry,2005,02:13-19.

[10] Hou Hong, Han Zhen-Yu. The PID Parameters Self-Turning Based on Relay Feedback [J]. Mechanical \& Electrical Engineering Technology, 2014, 07:63-65+187.

[11] Khalore, A.G., Date, T.N., Singh, S.. Performance Overview of Relay Feedback Tuning of PID Controller, India Conference (INDICON), 2012 Annual IEEE, vol., no., pp.198,204, 7-9 Dec. 2012

[12] Menani, S, Koivo, H.N. Automatic Tuning of Multivariable Controllers with Adaptive Relay Feedback, Decision and Control, 1996., Proceedings of the 35th IEEE Conference on , vol.4, no., pp.4695,4700 vol.4, 11-13 Dec 1996 